\title{
Monaural Azimuth Localization Using Spectral Dynamics of Speech
}

\author{
Roi Kliper ${ }^{1}$, Hendrik Kayser ${ }^{2}$, Daphna Weinshall ${ }^{1}$, Jörn Anemüller ${ }^{2}$ and Israel Nelken ${ }^{1}$ \\ ${ }^{1}$ Interdisciplinary Center for Neural Computation, Hebrew University of Jerusalem, Israel \\ ${ }^{2}$ Carl von Ossietzky University, Oldenburg, Germany \\ kliper@cs.huji.ac.il, hendrik.kayserduni-oldenburg.de
}

\begin{abstract}
We tackle the task of localizing speech signals on the horizontal plane using monaural cues. We show that monaural cues as incorporated in speech are efficiently captured by amplitude modulation spectra patterns. We demonstrate that by using these patterns, a linear Support Vector Machine can use directionality related information to learn to discriminate and classify sound location at high resolution. We propose a straightforward and robust way of integrating information from two ears: treating each ear as an independent processor and integrate the information at the decision level by doing that ambiguity is to a large extent resolved.
\end{abstract}

Index Terms: Speech localization, Amplitude modulation, Monaural

\section{Introduction}

The ability to perform speech and speaker localization is an important capacity of daily human communication. Accurate speech localization is a fundamental building block for advanced speech processing that handle problems such as stream segregation, source separation, source enhancement and denoising, ultimately producing increased speech intelligibility.

This paper employs Amplitude Modulation Spectra (AMS) patterns as representation of speech signals to perform monaural localization. While the efficacy of this representation for speech recognition has already been demonstrated, we show that these features can simultaneously capture location information included in a non-specific speech signal due to directiondependent filtering by the human head and pinnae. We show that the existence of this information allows for monaural localization. We propose and demonstrate the idea of treating the two ears as two parallel processors: each processing monaural information and reaching a hypothesis about the location of a given source. Integration between the two ears is achieved, in this view, at the level of the decision rather then at the level of analysis.

A sound wave generated by an external source is diffracted by the head and external ear (as well as other objects in the environment). The resulting changes in the temporal and intensive characteristics of the acoustical stimuli provide cues about the locus of the sound relative to the head. These localization cues have traditionally been divided to Binaural cues and Monaural cues and various researches have explored their relative efficacy in determining sound localization. Recent efforts has been devoted into understanding the integration of these different types of information, these efforts have spread from physiological research through psychoacoustic research to application derived research.

\subsection{Binaural localization}

Binaural hearing is a well established source of information for the localization of sound sources in space, building upon two distinctive properties of incoming sounds: interaural time differences (ITDs) and interaural level differences (ILDs). These differences arise from the fact that the two ears are separated by both space and an acoustically opaque mass (the head). Modeling of the processing of these cues build upon carefully constructed coincidence detectors, and are supported by both physiological and psychoacoustic findings.

While such models for sound localization are both elegant and robust these cues are limited in their efficient frequency band [1] and are presenting a major challenge to the integrative capabilities of the nervous system requiring very high accuracy in highly structured labeled lines. Furthermore, to the extent that the head and ears are symmetrical, interaural differences should provide no cue to the vertical location of a sound source on the median plane nor will it contribute to the resolution of the front back confusion. While, acknowledging these limitations, artificial system designers have favored models inspired by the binaural models for sound localization. Some challenge to the primacy of binaural localization cues have also been made (e.g. [2]); however, the role of monaural cues in sound localization is, to a great extent, only brought into focus in situations where binaural differences are nonexistent.

\subsection{Monoaural localization}

For a single ear, the changes in temporal and intensive characteristic of the signal are generally described by head related impulse response (HRIR), a generalization of which are Monaural Room Impulse Reposes (MRIRs). This parsimonious description captures all the directional influence a signal may have suffered on its way to the ear drum. For example, to some abstraction, the influence of the pinnae is to produce multiple paths to the ear canal, among them a direct path and a reflection from the structure of the pinnae. The addition of a direct path with a delayed path of the same signal produces a comb filtered spectrum containing a characteristic structure of peaks and notches. The pinnae thus, acts as a directionally dependent filter which strongly affects the HRIR at high frequencies.

Attempts to explain and exploit monaural cues for sound localization have been made by several researches. Generally speaking, these researches really on the differential way in which the HRIR effects the spectrum of the input signal and require some interaction and/or comparison between different spectral bands of the signal. In this respect, these researches compel to the fact that the signal appearing at the eardrum has no reference point but itself. A second requirement for monaural localization is some statistical model of the source signal or equivalently some restrictions to its statistical properties; direc- 
tional sensitivity in itself cannot, be exploited for general signal localization. This is since the system under general signal assumptions is underdetermined and may result in ambiguous localization as each sound signal can be manipulated such that it is perceived as coming from all other locations. Assuming that the source to be located is the statistically restricted set of speech signals is one such step.

In monaural localization of sound, and more specifically in monaural localization of speech one should bare in mind that intelligibility and localization often represent competing requirements where intelligibility requires minimum distortion while localization requires direction dependent distortions. Choosing AMS patterns as a representation of speech (see Section 2.3) highlights directional information while containing speech related information thus allowing a good balance of the intelligibility - distortion equilibrium.

\subsection{Physiological and psychoacoustic findings}

Psychoacoustic experiments have demonstrated monaural localization of a sound source on both the horizontal and the vertical planes [1]. In a recent paper Shub et al [3] have demonstrated the ability of human subjects to monaurally discriminate and classify different directions. Our experiments follow their paradigm and show that by employing a simple machine learning approach these results can be reproduced and surpassed (see Section 3.2).

Chase \& Young [4] explored how different acoustic localization cues are coded in the inferior Colliculus (IC). The rational was that the IC integrates binaural cues (ITD, ILD) and monaural cues (spectral information). Their results suggest that different cues converge to different degrees in different neurons: ITD and ILD are coded most strongly by spike rate while the spectral envelope of the signal is coded by the temporal pattern of the spikes. Localization in the vertical plane is often thought to be a purely monaural ability but recent psychophysical studies [5] have shown that both ears are used to the determine the vertical elevation, with the relative contribution of each ear varying with the horizontal location. Our binaural experiment successfully implements this idea for localization in the horizontal plane (see Section 3.2).

\section{Experimental setting}

\subsection{Data}

Experiments were carried out using a well known speech database (see Section 2.1.1) and a database of HRIRs (see Section 2.1.2), these together provide a rich but reproducible setting while allowing control over the experimental conditions.

\subsubsection{Speech}

Speech data was taken from the TIMIT Speech corpus [6] which provides recordings of 630 different speakers each reading ten phonetically rich sentences recorded at sampling rate of $16 \mathrm{kHz}$. The segmentation into train and test data was adopted from the corpus. Both of the sets were further divided into direction specific subsets sampled randomly from the dataset and concatenated. No intersection between any of the subsets was allowed.

\subsubsection{Head-related impulse responses}

A database of head-related impulse responses [7] was employed to introduce directional characteristics to the speech data. The database provides, among others, HRIRs measured on an head and torso simulator equipped with in-ear microphones under anechoic conditions. The azimuthal resolution is $5^{\circ}$ covering the full azimuthal plane. HRIRs from an elevation angle of $0^{\circ}$ (no elevation) and a radius of $3 \mathrm{~m}$ were taken. The initial delay contained in the HRIRs was removed and the remaining impulse response was cut to a length of $10 \mathrm{~ms}$.

The audio data was convolved with the MRIR corresponding to a specific direction, resulting in monaural sound signals containing the input to either the left or the right ear. All data was scaled to unit variance to compensate for overall level differences between signals according to different directions. For experiments carried out in the presence of diffuse noise, pink random noise was generated artificially. The noise signal was convolved with each direction's HRIR from the full circle except for the one the target speech source was impinging from well approximating an isotropic noise field. After convolution, the noise was added to the directional speech source with the desired SNR. The coordinate system for the azimuth angles is relative to the center of the horizontal plane and is $0^{\circ}$ in front of the head $-90^{\circ}$ and $90^{\circ}$ in front of the left and right ear.

\subsection{Classification}

Linear support vector machines [8] (SVMs) were employed to conduct training of discriminative models using AMS features. The task consist of either binary classification, where a model was trained to discriminate between the directions of two speech sources, or a multi-class classification task, where a model was trained to estimate the absolute direction of an impinging speech source in a $1 \mathrm{vs}$. all approach given a set of more than two directions.

\subsection{AMS features and their extraction}

Following the structure of the temporal envelope has shown to be crucial in human and machine recognition of speech. As a consequence, low modulation frequencies were employed for several tasks in speech processing and acoustic scene analysis. These features deliver a robust and generalized representation of speech signals and are known to be largely invariant to speaker and channel variations such as pitch and spectral distortions in the input signal. They showed to be robust representation of speech even in challenging conditions, e.g. in speech detection experiments [9]. The features that are employed here are based on AMS [10] and are calculated as follows (cf. Figure 1):

The amplitude modulation spectrogram analyzes sound signals with respect to their modulation content by decomposing them into time, frequency and modulation-frequency components. It is computed by first extracting the spectral envelopes

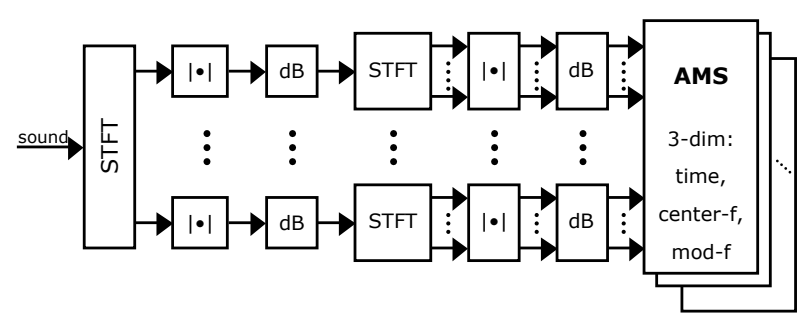

Figure 1: AMS calculation flow: Extraction of amplitude modulation features generates a 3-dimensional representation of the input. For each 100 ms signal segment a $256 \times 9$ pattern is extracted, overlap of neighboring patterns is $50 \mathrm{~ms}$. 
of the acoustic signal via an short-term Fourier Transformation (STFT) with a $32 \mathrm{~ms}$ Hamming window with a shift of $2 \mathrm{~ms}$ followed by computation of the log-energy. To extract modulation energy in each spectral band, another STFT is applied employing a $100 \mathrm{~ms}$ Hamming window with a shift of $50 \mathrm{~ms}$.

Finally, the log-energy is computed again and the DCcomponent of the modulation spectrum, containing the acoustic frequency spectrum of the input signal, and the first acoustic frequency component of the resulting AMS pattern are removed to disregard spectral properties of the signal. Modulation frequencies above $100 \mathrm{~Hz}$ are also removed. The resulting AMS spectra for each time frame span 256 frequency bands from $32-8000 \mathrm{~Hz}$ and modulation frequency bands covering the range from $20 \mathrm{~Hz}$ to $100 \mathrm{~Hz}$ with a resolution of $10 \mathrm{~Hz}$. The employed range patterns lie above modulation frequencies used in speech recognition and detection.

\section{Experiments and results}

In the following we present first, experiments of a discrimination task which is a popular task in psychoacoustics used to evaluate minimum audible difference. We then show results of multi-direction classification experiments under different noise conditions. Finally, results of a straightforward winner takes all formalism of binaural integration are presented.

\subsection{Minimum audible angle difference}

In this experiment a set of models for binary classification was trained to discriminate between two sound sources $s_{1}$ and $s_{2}$ impinging from different directions. $s_{1}$ (target) was located at the positions $0^{\circ}$ to $-180^{\circ}$ in steps of $-60^{\circ}$ while the angular position of $s_{2}$ (reference) is varied with a resolution of $5^{\circ}$ on the same half circle. The results are shown in Figure 2.

The superiority of the contralateral monaural cues over the ipsilateral can be read from the consistent improved accuracy of the right ear on the left hemisphere (blue line above red line in e.g. Figure 2 B). Contralateral cues achieve a nearly perfect performance discriminating the target from references around it at a resolution of $5^{\circ}$. This makes sense as heavier distortion has been introduced in the contralateral case thus allowing better directional sensitivity. We note that, as we normalized the energy the eardrum, our results cannot be accounted for by referring to level differences (which will appear in real scenarios). One can also note (1) The appearance of moderate front to back confusion in the cases where $s_{1}$ is located exactly in front or behind the listener (see Figure 2 A,D). (2) Increased performance in the frontal half space. (compare Figure 2 B,C). Similar discrimination characteristics can be found in psychoacoustic monaural localization experiments carried out in [3], however while their experiment was done with stimuli ten times longer our results surpass theirs.

\subsection{Localization of sound sources in the presence of noise and the integration of two ears}

In the multi-class classification experiment we first evaluated the influence of averaging the features over classification performance. For that, a sliding average over time was applied to the AMS patterns before models were trained. Averaging was conducted with a sliding window of length ranging from $0 \mathrm{~s}$ (no averaging) to $5 \mathrm{~s}$ in steps of $0.25 \mathrm{~s}$. Furthermore robustness against diffuse pink noise was investigated: noise was introduced as described in Section 2.1 with an SNR varying from $10 \mathrm{~dB}$ to $60 \mathrm{~dB}$ in steps of $10 \mathrm{~dB}$, and infinity.

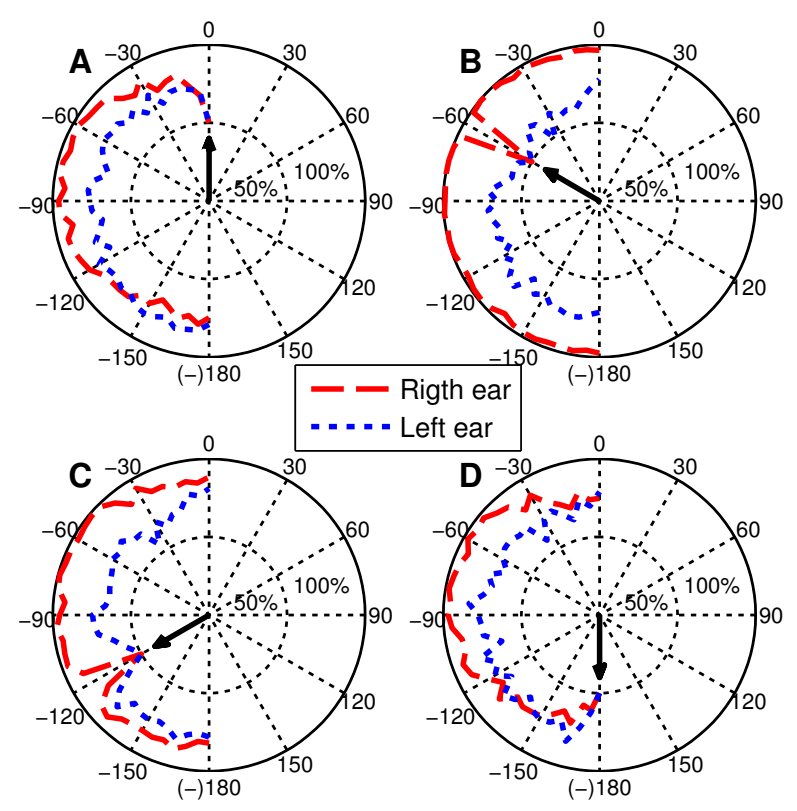

Figure 2: Binary classification: The accuracies gained by the Right (contralateral) ear are shown in dotted blue and by the Left (ipsilateral) ear are shown in dashed red. Four target signals where classified against a reference signal drawn one at a time from the left hemisphere at a $5^{\circ}$ resolution. Performance in terms of testing accuracy is shown on the radial axis. Position of reference signals are shown on the circular axis. Target signal is pointed by a black arrow.

Figure 3 displays the results of this experiment for 11 directions distributed symmetrically and equally spaced around $0^{\circ}$ between $-150^{\circ}$ and $150^{\circ}$. The accuracies gained by the left and the right ear independently, in addition to the the performance achieved by the integration of information from both ears are shown. For each ear, one model was trained and tested on one condition given by the SNR and length of averaging. Different noise condition appear in Figure $3 \mathrm{~A}-\mathrm{G}$ and show a clear dependency on noise condition ranging from chance level $(9 \%)$ at SNR $10 \mathrm{~dB}$ to $60 \%$ for a single ear and $81.2 \%$ for the combination of to ears where no noise is present (inf). Average accuracy as dependent on the averaging length is read against the $x$-axis in each figure and shows a global maximum at around $1 \mathrm{~s} \mathrm{sug-}$ gesting that longer averaging windows are erasing localization information. Integration of the information from the two ears was done in a winner takes all manner where the more confident ear was taken as the reliable one. Confidence was assumed to be positively correlated to the margin from the model's separating hyperplane. Integration of the two ears achieves up to $20 \%$ boost in average accuracy.

Besides the overall average testing accuracy the confusion between different directions is a significant figure of merit. The confusion matrices obtained for a complete circle $\left(-180^{\circ}\right.$ to $150^{\circ}$ in steps of $30^{\circ}$ ) from the left and the right ear and from the integration of both ears are shown in Figure 4. Clear preference for the contralateral ear can be read in Figure 4 A B. The Ipsilateral ear regains classification efficiency only when angular distance is larger then $60^{\circ}$ (allowing for directional cues to come to effect). Integration on the information from the two ears solves most of the confusion, leaving a slightly increases confusion around the center supposably due to noise in the confidence of the classifiers. 

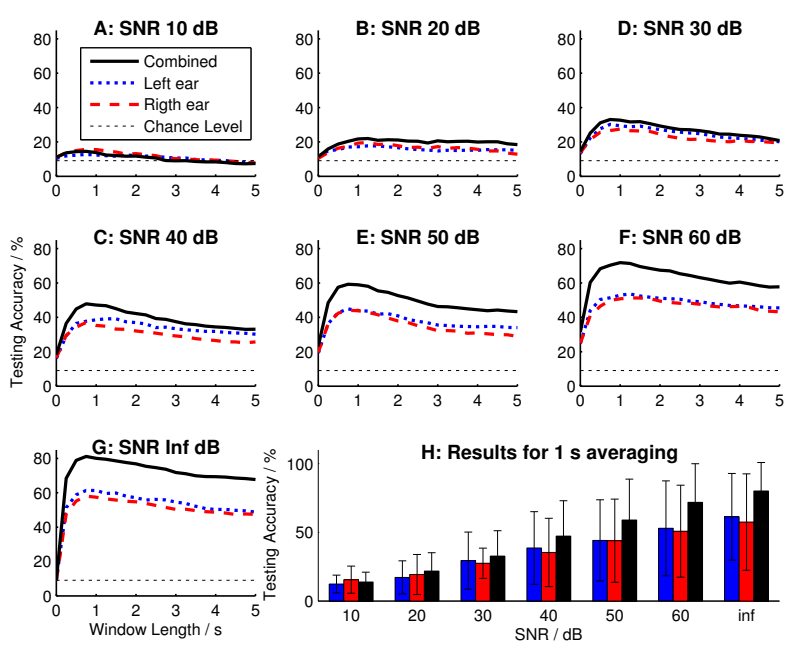

Figure 3: Multi-class classification at different SNRs: A-G: Performance of the localization on 11 directions for different SNRs and averaging times of the features. Blue and red lines denote the accuracy achieved by the single ears, black the performance of the integrated approach. H: Average and standard deviation of accuracy for each SNR.

\section{Discussion and conclusions}

We have demonstrated that amplitude modulation spectra patterns in modulation frequencies above those commonly used for other speech applications are efficient in monaural speech localization. Our results suggest that under clean and moderate noise conditions, accurate speech localization can be achieved using the information obtained by a single ear, without distorting intelligibility related information. The experiments showed a maximal performance for an integration time of around $1 \mathrm{~s}$, which corresponds to the choice of parameters in monaural localization experiments conducted by Shub et al. Keeping in mind, that the analysis of the stimuli was done using a rather technical approach, further investigation incorporating auditory models as a preprocessing stage is a natural step. While monaural localization was proven to be feasible, the integration of information processed parallel in the two ears is highly beneficial as each ear performs better on the contralateral hemisphere. Relating to physiological research we hypothesize that the IC may host such an integration mechanism.

This success in speech source localization highlight the potential use of the described method in the context of other speech processing applications such as source separation, signal to noise ratio estimation, and noise reduction. Drawing to the design of artificial systems, the implementation of such an approach in ear-worn hearing assistive systems is an interesting application as it does not necessarily demand for a technically costly cross-linking in the case of a bilateral means.

\section{Acknowledgements}

Support by the DFG Collaborative Research Centre SFB/TRR 31 The Active Auditory System acknowledged by H.K. \& J.A.

\section{References}

[1] J. Middlebrooks and D. Green, "Sound localization by human listeners," Annual Review of Psychology, vol. 42, no. 1, pp. 135-159, 1991.
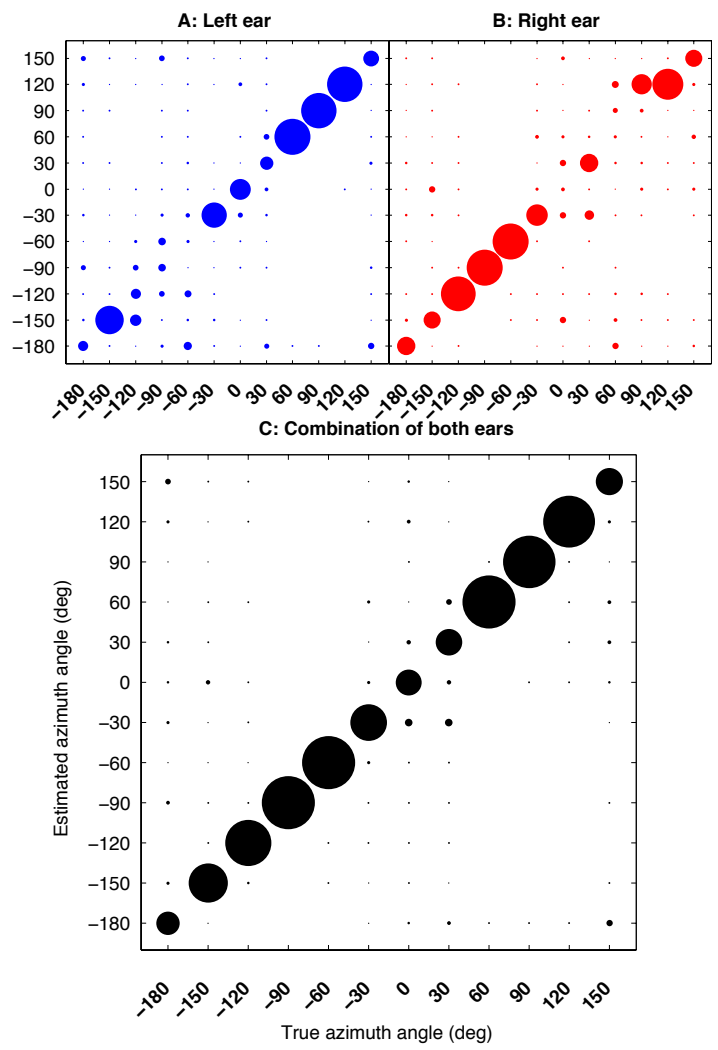

Figure 4: Confusion matrix for multi-direction classification: Confusion matrices for single ears (upper row) and after integration of both ears for the clean condition and averaging window length of $1 \mathrm{~s}$. Coincidence of target and reference is given by the radius of the circle.

[2] H. Fisher and S. Freedman, "The role of the pinna in auditory localization." Journal of Auditory research, 1968.

[3] D. Shub, S. Carr, Y. Kong, and H. Colburn, "Discrimination and identification of azimuth using spectral shape," The Journal of the Acoustical Society of America, vol. 124, p. 3132, 2008.

[4] S. Chase and E. Young, "Cues for sound localization are encoded in multiple aspects of spike trains in the inferior colliculus," Journal of neurophysiology, vol. 99, no. 4, p. 1672, 2008.

[5] P. Hofman and A. Van Opstal, "Binaural weighting of pinna cues in human sound localization," Experimental brain research, vol. 148, no. 4, pp. 458-470, 2003.

[6] J. S. Garofolo, L. F. Lamel, W. M. Fisher, J. G. Fiscus, D. S. Pallett, and N. L. Dahlgren, DARPA TIMIT Acoustic Phonetic Continuous Speech Corpus CDROM, U.S. Dept. of Commerce NTIS, Gaithersburg, MD, 1990.

[7] H. Kayser, S. Ewert, J. Anemüller, T. Rohdenburg, V. Hohmann, and B. Kollmeier, "Database of multichannel in-ear and behindthe-ear head-related and binaural roomimpulse responses," EURASIP Journal on Advances in Signal Processing, p. 10, 2009.

[8] R.-E. Fan, K.-W. Chang, C.-J. Hsieh, X.-R. Wang, and C.-J. Lin, "LIBLINEAR: A library for large linear classification," Journal of Machine Learning Research, vol. 9, pp. 1871-1874, 2008.

[9] J. Bach, B. Kollmeier, and J. Anemüller, "Modulation-based detection of speech in real background noise: Generalization to novel background classes," in Acoustics Speech and Signal Processing (ICASSP), 2010 IEEE International Conference on. IEEE, 2010, pp. 41-44.

[10] B. Kollmeier and R. Koch, "Speech enhancement based on physiological and psychoacoustical models of modulation perception and binaural interaction," The Journal of the Acoustical Society of America, vol. 95, p. 1593, 1994. 